\title{
Sugar content analysis and expression profiling of sugar related genes in contrasting Strawbeny (Fragaria $\times$ ananassa) cultivars
}

\author{
Ashokraj Shanmugam • Mohammad Rashed Hossain • Sathishkumar Natarajan • Hee-Jeong Jung • Jae-Young Song • \\ Hoy-Taek Kim • Ill-Sup Nou
}

Received: 9 June 2017 / Revised: 27 June 2017 / Accepted: 30 June 2017

(C) Korean Society for Plant Biotechnology

\begin{abstract}
Fragaria $\times$ ananassa, a strawberry evolved from hybridization between $F$. virginiana and $F$. chiloensis, is a globally cultivated and consumed fruit crop valued for its flavor and nutritional value. Flavor and quality of fruits are determined by factors such as sugars and organic acids present during fruit development. These characteristics are highly subjective in different genotypes and affected by various environmental factors. In this study, we analyzed contents of major sugar compounds including fructose, glucose and sucrose by HPLC analysis in four cultivars namely, Maehyang, Seolhyang, Festival and Sweet Charlie. We identified 55 genes related to fructose, glucose, sucrose and soluble sugar regulation whose expression were analyzed in four cultivars at three developmental stages of the fruit namely, green, white and ripened stages. Expression of these genes across these progressive fruit developmental stages varied among cultivars. Among the 55 genes, genes FaFru3, FaSuc11 and FaGlu8 revealed differential patterns of expression along developmental stages of the fruit in high and low sugar-containing genotypes, respectively and may be putative candidates for sugar content in strawberries. Expression of genes are discussed with regard to corresponding sugar content in these genotypes. Further analysis and application of these genes may be valuable in developing high sugar containing cultivars via marker-assisted breeding.
\end{abstract}

A. Shanmugam · M. R. Hossain · S. Natarajan · H.-J. Jung ·

Ill-Sup Nou $(\varangle)$

Department of Horticulture, Sunchon National University,

Suncheon 57922, Republic of Korea

e-mail: nis@sunchon.ac.kr

J.-Y. Song

Department of Crop Science, Chungbuk National University, Chengju 28644, Republic of Korea

H.-T. Kim $(\square)$

University-Industry Cooperation Foundation, Sunchon National

University, Suncheon 57922, Republic of Korea

e-mail: htkim@sunchon.ac.kr
Keywords $\quad$ Fragaria $\times$ ananassa, Sugar content, Sugar-related genes, Expression profiling

\section{Introduction}

Fragaria $\times$ ananassa, the cultivated strawberry evolved by hybridization between $F$. virginiana and $F$. chiloensis, is globally cultivated fruit crop (Molina-Hidalgo et al. 2013; Bestfleisch et al. 2014). It is the rich source of health-promoting phytonutrients (e.g., vitamin C, vitamin B-6, niacin, riboflavin, pantothenic acid, vitamin-E and folate, etc.), flavonoid poly phenolic antioxidants (e.g., lutein, zeaxanthin, and beta-carotene etc.) and minerals (e.g., potassium, manganese, fluorine, copper, iron and iodine) (Wang, Shiow Y., Lin 2000). The consumption of strawberry is known to lower the risk of inflammation, hypertension, cardiovascular diseases and cancer (Basu et al. 2014). Strawberry is thus widely consumed, fresh or in processed forms, making it one of the economically and commercially more important fruit crop and the most studied berries from the agronomic, genomic, and nutritional characteristics (Giampieri et al. 2013). Many conventional and biotechnological approaches were applied to develop the stress tolerance, high quality and flavored strawberry cultivars (Molina-Hidalgo et al. 2013; Bestfleisch et al. 2014).

The sweet flavor and quality of fruits are determined by factors such as sugars and organic acids (Kallio et al. 2000). Due to its inherent chemical composition, strawberry is naturally sensitive fruit rendering the quality of the fruits being affected by environmental factors. Moreover, ripening of strawberry fruits is complex process which includes the participation of a plethora of hormones, sugars and other volatile compounds (Kafkas et al. 2007).

The sweetness, an important sensory quality of the fruit is largely determined by the total soluble sugars and major carbohydrates such as glucose, sucrose and fructose etc. 
The contents of these sugars and carbohydrates are variable based on the genotypes and environmental factors (MacíasRodríguez et al. 2002; Gündüz and Özdemir 2014). Of which, glucose is particularly abundant carbohydrate whereas sucrose is present at low concentration in many strawberry cultivars (Basson et al. 2010; Gündüz and Özdemir 2014).

Sucrose, glucose and fructose are the three major sugars which increases significantly in accordance with fruit development and accounts of $99 \%$ of the total sugar content (Jia et al. 2013). Among these three major sugars, sucrose content is highly responsible for fruit development whereas glucose and fructose was not much influenced during fruit development. Sucrose plays an important role in fruit ripening by regulation of ripening-related genes (Jia et al. 2013). In addition, the sucrose and glucose induces the biosynthesis of ABA which is regulating the strawberry fruit development by regulating the ripening related genes (Jia et al. 2013; Jia et al. 2016). Therefore, the soluble sugars and major carbohydrates including sucrose, glucose and fructose are among the major determinants of the growth, development, sweetness and overall quality of the strawberry fruits. Our study thus focuses on broadening the understanding of the roles of sugar regulatory and biosynthetic genes in the developmental process of strawberry fruits via analyzing the contents of sugar and the relative expression of total sugar related genes in contrasting strawberry cultivars.

\section{Materials and Methods}

Collection of strawberry cultivars

For this study, fruit materials of four strawberry cultivars namely, Maehyang, Seolhyang, Festival and Sweet Charlie were grown in two different research facility; Suncheon National University and Chungnam Agricultural Research \& Extension, South Korea. The sugar content in fruits of four cultivars were measured by Pocket Refractometer PAL-1 (Atago, Japan) in the year 2016 and the data is compared with previous report by Kim al., (2009) as shown in Table 1.
HPLC analysis of sugar content

Fruit samples were collected at different maturation stage and stored at $-80^{\circ} \mathrm{C}$. The collected samples were powdered and dissolved in distilled water at the ratio of $2 \mathrm{~g}$ in 20 $\mathrm{mL}$ ratio. Then the solution was filtered through $0.45 \mu \mathrm{m}$ filter. This filtrate was collected and used for individual sugar analysis by HPLC.

Gene identification and characterization

The sugar related genes were manually identified from Fvesca_V1.0_genemark_hybrid annotation available from the Strawberry garden database (http://strawberry-garden. kazusa.or.jp/). The gene sequences were blasted against the Fragaria $\times$ ananassa draft genome (FANhybrid r1.2_cds) available from the same database. Conserved functional domains were analyzed for identified genes by using CDD (Conserved Domain Database, http://www.ncbi.nlm.nih.gov/ Structure/cdd/cdd.shtml) (Marchler-Bauer et al. 2015) database. The gene description, and gene ontology (GO) were carried by using Blast2Go (https:// www.blast2go.com) for the sugar related genes (Natarajan et al. 2016).

\section{Expression analysis}

Samples of leaf and three stages of fruit development; green, white and ripening stages were used for total RNA isolation by using RNeasy mini kit (Qiagen, USA) based on manufacturer's protocol. RNAse free DNase (Qiagen, USA) was added to remove genomic DNA contamination. The cDNA was synthesized from isolated RNA samples by using Superscript III ${ }^{\circledR}$ First-strand Synthesis Supermix kit (Invitrogen, USA). ND-1000 Spectrophotometer and NanoDrop v3.7 software were used for quantification and purity determination (NanoDrop Technologies, USA).

Gene-specific primers were designed by using the primer3 software in coding DNA sequences to perform RT-PCR and Real-time PCR (qPCR). We used the actin as the reference gene for expression analysis (Amil-Ruiz et al. 2013; Galli

Table 1 Sugar content of four strawberry cultivars measured by Pocket Refractometer PAL-1

\begin{tabular}{lccr}
\hline \multirow{2}{*}{ Cultivars } & \multicolumn{3}{c}{ Fruit Soluble Sugar Content (SSC) (Brix) } \\
\cline { 2 - 4 } & Kim, et al. (2009) & $\begin{array}{c}\text { Chungnam Agriculture } \\
\text { and Extension (2016) }\end{array}$ & $\begin{array}{r}\text { Sunchon National University } \\
(2016)\end{array}$ \\
\hline Maehyang & 12.7 & 9.1 & 10.9 \\
Seolhyang & 12.8 & 9.1 & 10.1 \\
Festival & - & 6.1 & 8.7 \\
Sweet Charlie & 6.4 & 4.3 & 7.9 \\
\hline
\end{tabular}


et al. 2015). RT-PCR was performed by using $50 \mathrm{ng}$ cDNA $(1 \mu \mathrm{L}), 20$ pmol primer pairs $(2 \mu \mathrm{L})$, Emerald master mix ( $8 \mathrm{~L}$ ), and $\mathrm{H}_{2} \mathrm{O}$ with total volume of $20 \mu \mathrm{L}$. Thermal cycler was set at 30 cycles of $60^{\circ} \mathrm{C}$ for $30 \mathrm{~s}$ and $72^{\circ} \mathrm{C}$ for 45 $\mathrm{s}$, followed by a final extension at $72^{\circ} \mathrm{C}$ for $5 \mathrm{~min}$. The PCR products were visualized on a $2 \%$ agarose gel. For qRT-PCR, $1 \mu \mathrm{L}$ cDNA with $10 \mu \mathrm{L}$ iTaq SYBR Green Super-mix with ROX (California, USA) was used with three step amplification (annealing temperature $60^{\circ} \mathrm{C}$ ) and melting peak with three biological replicates. Amplification, detection, and data analysis were carried out using a Light Cycler ${ }^{\circledR} 96$ Instrument (Roche Diagnostics, United States). Single melting peak was considered for the primer specificity (Shanmugam et al. 2016; Vijayakumar et al. 2016).

\section{Results}

Sugar content analysis in different strawberry cultivars

The HPLC analysis was used to analysis the sugar content in different cultivars. HPLC analysis showed Maehyang and Seolhyang as high sugar content cultivar (12.23 and $14.10 \mathrm{~g} / 100 \mathrm{~g}$, respectively) whereas Festival and Sweet Charlie as low sugar content (5.05 and $4.78 \mathrm{~g} / 100 \mathrm{~g}$, re- spectively) cultivar based on their fructose, glucose, sucrose and total sugar content (Table 2). Overall, highest total sugar content is present in the cultivar Seolhyang $(14.10 \mathrm{~g} / 100 \mathrm{~g})$ followed by Maehyang (12.23 g/100g). Among the sugars detected, Seolhyang contained more fructose and glucose (4.22 and $4.30 \mathrm{~g} / 100 \mathrm{~g}$, respectively) compared to the other high sugar containing cultivar Maehyang and to low sugar containing cultivars Festival and Sweet Charlie where the content of fructose and glucose ranged between $1.95-2.41$ g/100g (Table 2 and Fig. S1). Unlike, fructose and glucose, sucrose content was the highest in Meahyang ( $7.80 \mathrm{~g} / 100 \mathrm{~g})$ compared to Seolhyang (5.22 g/100g) (Table 2 and Fig. S1). The other sugar, Maltose is detected at very trace level in the high sugar containing genotypes Maehyang and Seolhyang whereas this was not at all detected in low sugar containing genotypes.

\section{Identification of sugar-related genes}

In this study, we identified 6 fructose related genes, 16 glucose-related genes, 18 sucrose-related genes and 15 total soluble sugar-related genes (55 in total) from Fvesca_V1.0 genemark hybrid annotated available from the Strawberry garden database (http://strawberry-garden.kazusa.or.jp/) as shown with their description (Table 3-6). The functional

Table 2 Sugar content in the four Strawberry cultivars measured by HPLC

\begin{tabular}{lccccc}
\hline \multirow{2}{*}{ Cultivars } & \multicolumn{5}{c}{ Sugars } \\
\cline { 2 - 6 } & Fructose & Glucose & Sucrose & Maltose & Total \\
\hline Maehyang & 2.41 & 2.01 & 7.80 & 0.007752 & 12.23 \\
Seolhyang & 4.22 & 4.30 & 5.22 & 0.358124 & 14.10 \\
Festival & 2.39 & 2.07 & 0.59 & Not detected & 5.05 \\
Sweet Charlie & 2.07 & 1.95 & 0.76 & Not detected & 4.78 \\
\hline g- Gram & & & & &
\end{tabular}

Table 3 Fructose-related genes identified in Fragaria $\times$ ananassa

\begin{tabular}{|c|c|c|c|c|}
\hline Name & Gene id & $\begin{array}{l}\text { CDS length } \\
\text { (bp) }\end{array}$ & $\begin{array}{l}\text { Protein } \\
\text { length }(\mathrm{aa})\end{array}$ & Description* \\
\hline FaFru1 & FANhyb_icon00023264_a.1.g00001.1 & 428 & 143 & $\begin{array}{l}\text { pyrophosphate--fructose } 6 \text {-phosphate } \\
\text { 1-phosphotransferase subunit beta-like }\end{array}$ \\
\hline FaFru2 & FANhyb_rscf00002565.1.g00001.1 & 1755 & 584 & fructose-bisphosphate aldolase chloroplastic \\
\hline FaFru3 & FANhyb_rscf00000635.1.g00002.1 & 867 & 288 & glutamine--fructose-6-phosphate aminotransferase \\
\hline FaFru4 & FANhyb_rscf00002698.1.g00001.1 & 1390 & 462 & chloroplast fructose-1,6-bisphosphatase I \\
\hline FaFru5 & FANhyb_rscf00000435.1.g00002.1 & 1296 & 431 & $\begin{array}{l}\text { pyrophosphate--fructose 6-phosphate } \\
\text { 1-phosphotransferase subunit alpha }\end{array}$ \\
\hline FaFru6 & FANhyb_rscf00001420.1.g00002.1 & 1053 & 350 & $\begin{array}{l}\text { fructose-bisphosphate aldolase cytoplasmic } \\
\text { isozyme-like isoform } \mathrm{X} 1\end{array}$ \\
\hline
\end{tabular}

bp - base pair, aa - amino acid. *BLAST search against NCBI NR database using BLAST2GO. 
Table 4 Glucose-related genes identified in Fragaria $\times$ ananassa

\begin{tabular}{|c|c|c|c|c|}
\hline Name & Gene id & $\begin{array}{l}\text { CDS length } \\
\text { (bp) }\end{array}$ & $\begin{array}{c}\text { Protein } \\
\text { Length (aa) }\end{array}$ & Description* \\
\hline FaGlu1 & FANhyb_rscf00000042.1.g00027.1 & 1647 & 548 & glucose-6-phosphate 1-dehydrogenase chloroplastic \\
\hline FaGlu2 & FANhyb_rscf00000135.1.g00004.1 & 1449 & 482 & UDP-glucose 6-dehydrogenase 1 \\
\hline FaGlu3 & FANhyb_rscf00001884.1.g00003.1 & 1188 & 395 & $\begin{array}{l}\text { glucose-6-phosphate phosphate translocator } \\
\text { chloroplastic-like }\end{array}$ \\
\hline FaGlu4 & FANhyb_rscf00000034.1.g00011.1 & 1443 & 480 & UDP-glucose 6 -dehydrogenase 1 \\
\hline FaGlu5 & FANhyb_rscf00000166.1.g00017.1 & 1581 & 526 & $\begin{array}{l}\text { glucose-1-phosphate adenylyltransferase large subunit } \\
\text { chloroplastic amyloplastic }\end{array}$ \\
\hline FaGlu6 & FANhyb_rscf00000097.1.g00020.1 & 1788 & 595 & glucose-6-phosphate 1- chloroplastic \\
\hline FaGlu7 & FANhyb_rscf00001267.1.g00002.1 & 1461 & 486 & plastidic glucose transporter 4 \\
\hline FaGlu8 & FANhyb_rscf00000021.1.g00018.1 & 1761 & 586 & glucose-6-phosphate cytosolic \\
\hline FaGlu9 & FANhyb_rscf00000482.1.g00001.1 & 1566 & 521 & $\begin{array}{l}\text { glucose-1-phosphate adenylyltransferase large subunit } \\
\text { 1-like }\end{array}$ \\
\hline FaGlu10 & FANhyb_rscf00002113.1.g00001.1 & 2424 & 807 & glucose-6-phosphate 1- chloroplastic-like \\
\hline FaGlu11 & FANhyb_rscf00000504.1.g00004.1 & 1764 & 587 & glucose-6-phosphate 1- chloroplastic \\
\hline FaGlu12 & FANhyb_rscf00000013.1.g00018.1 & 1443 & 480 & UDP-glucose 6-dehydrogenase 5 \\
\hline FaGlu13 & FANhyb_rscf00000040.1.g00031.1 & 1041 & 346 & probable plastidic glucose transporter 2 \\
\hline FaGlu14 & FANhyb_rscf00001636.1.g00003.1 & 1059 & 352 & UTP--glucose-1-phosphate uridylyltransferase \\
\hline FaGlu15 & FANhyb_rscf00000538.1.g00003.1 & 1047 & 348 & UDP-glucose 4-epimerase GEPI48-like \\
\hline FaGlu16 & FANhyb_rscf00002773.1.g00002.1 & 687 & 228 & $\begin{array}{l}\text { bifunctional UDP-glucose 4-epimerase and UDP-xylose } \\
\text { 4-epimerase } 1\end{array}$ \\
\hline
\end{tabular}

bp - base pair, aa - amino acid. *BLAST search against NCBI NR database using BLAST2GO.

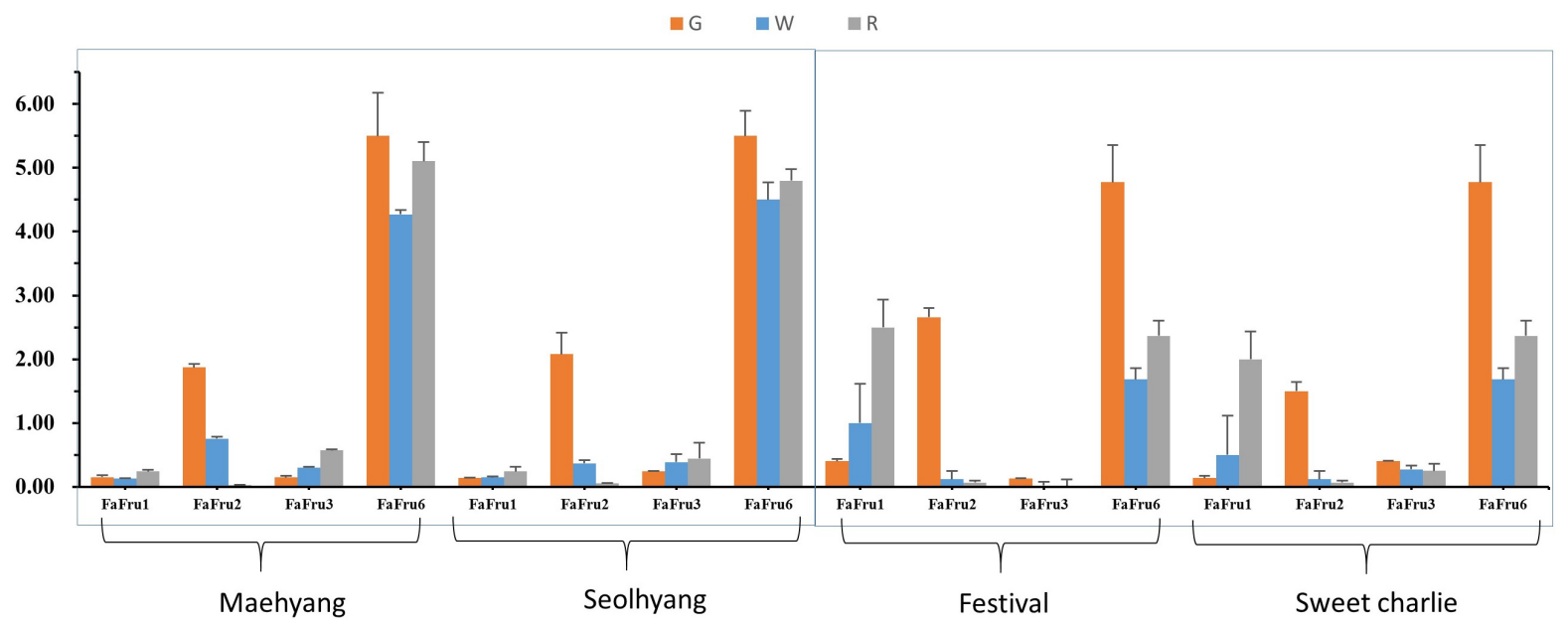

Fig. 1 Expression profiling of fructose related genes in contrasting strawberry cultivars

domains were determined for the identified genes and are shown in (Supplementary Table 1-4). Further, gene ontology and functional analysis were determined for the identified sugar-related genes in strawberry (Supplementary Table 5-8 and Fig. S2).

Expression profiling of Fructose related genes

Among six fructose related genes, expression of four genes were expressed in the four strawberry cultivars (Fig. 1). The other two genes were not expressed and hence, was not included in our results. The results are shown in (Fig. 1). The genes showed variable patterns of expression during different developmental stages in different cultivars. Only the gene FaFru 3 showed contrasting patterns of expression between high and low sugar containing cultivars. It's expression increased with the progress in the maturation stages fruit in both the high sugar containing cultivars, but 
Table 5 Sucrose-related genes identified in Fragaria $\times$ ananassa

\begin{tabular}{clccl}
\hline Name & \multicolumn{1}{c}{ Gene id } & $\begin{array}{c}\text { CDS length } \\
(\mathrm{bp})\end{array}$ & $\begin{array}{c}\text { Protein } \\
\text { length (aa) }\end{array}$ & \multicolumn{1}{c}{ Description* } \\
\hline FaSuc1 & FANhyb_rscf00001083.1.g00004.1 & 2271 & 756 & probable galactinol--sucrose galactosyltransferase 1 \\
FaSuc2 & FANhyb_rscf00000994.1.g00002.1 & 1983 & 659 & sucrose transport SUC3 \\
FaSuc3 & FANhyb_rscf00000541.1.g00007.1 & 2520 & 839 & probable sucrose-phosphate synthase 3 \\
FaSuc4 & FANhyb_rscf00007596.1.g00001.1 & 970 & 322 & sucrose synthase 2 \\
FaSuc5 & FANhyb_rscf00001056.1.g00003.1 & 780 & 259 & sucrose transport SUC2-like \\
FaSuc6 & FANhyb_rscf00004885.1.g00001.1 & 1340 & 445 & sucrose synthase \\
FaSuc7 & FANhyb_rscf00000111.1.g00005.1 & 3006 & 1001 & probable sucrose-phosphate synthase 1 \\
FaSuc8 & FANhyb_rscf00000334.1.g00004.1 & 2421 & 806 & sucrose synthase \\
FaSuc9 & FANhyb_rscf00000021.1.g00004.1 & 1488 & 495 & sucrose transport -like \\
FaSuc10 & FANhyb_rscf00000021.1.g00005.1 & 951 & 316 & sucrose transport SUC2-like \\
FaSuc11 & FANhyb_rscf00000142.1.g00011.1 & 2211 & 736 & probable galactinol--sucrose galactosyltransferase 2 \\
FaSuc12 & FANhyb_icon00036727_a.1.g00001.1 & 335 & 111 & sucrose transport SUC2-like \\
FaSuc13 & FANhyb_rscf00000011.1.g00010.1 & 2622 & 873 & probable galactinol--sucrose galactosyltransferase 6 \\
FaSuc14 & FANhyb_rscf00000011.1.g00003.1 & 5379 & 1792 & probable sucrose-phosphate synthase 1 \\
FaSuc15 & FANhyb_rscf00001350.1.g00002.1 & 2991 & 996 & probable sucrose-phosphate synthase 4 \\
FaSuc16 & FANhyb_rscf00000207.1.g00012.1 & 2454 & 817 & sucrose synthase 7 \\
FaSuc17 & FANhyb_icon00016073_a.1.g00001.1 & 569 & 189 & galactinol--sucrose galactosyltransferase-like \\
FaSuc18 & FANhyb_rscf00001543.1.g00001.1 & 3195 & 1064 & sucrose transport SUC2-like \\
\hline
\end{tabular}

bp - base pair, aa - amino acid. *BLAST search against NCBI NR database using BLAST2GO.

Table 6 Soluble sugar-related genes identified in Fragaria $\times$ ananassa

\begin{tabular}{ccccl}
\hline \multirow{2}{*}{ Name } & \multicolumn{1}{c}{ Gene id } & $\begin{array}{c}\text { CDS length } \\
(\mathrm{bp})\end{array}$ & $\begin{array}{c}\text { Protein } \\
\text { length (aa) }\end{array}$ & \multicolumn{1}{c}{ Description* $^{*}$} \\
\hline FaSug1 & FANhyb_rscf00004633.1.g00001.1 & 1036 & 344 & sugar transport 13-like \\
FaSug2 & FANhyb_rscf00003570.1.g00001.1 & 1611 & 536 & sugar transport 13-like \\
FaSug3 & FANhyb_rscf00004805.1.g00001.1 & 955 & 317 & sugar transport 13-like \\
FaSug4 & FANhyb_rscf00000050.1.g00021.1 & 1473 & 490 & sugar transporter ERD6-like 16 isoform X1 \\
FaSug5 & FANhyb_rscf00000071.1.g00017.1 & 1734 & 577 & UDP-sugar pyrophosphorylase \\
FaSug6 & FANhyb_rscf00000006.1.g00036.1 & 1431 & 476 & sugar transport 14 \\
FaSug7 & FANhyb_rscf00000046.1.g00026.1 & 1560 & 519 & sugar carrier C \\
FaSug8 & FANhyb_rscf00000069.1.g00020.1 & 1566 & 521 & sugar transport 10-like \\
FaSug9 & FANhyb_rscf00001892.1.g00002.1 & 1266 & 421 & sugar transporter ERD6-like 3 \\
FaSug10 & FANhyb_icon00015561_a.1.g00001.1 & 364 & 121 & sugar transporter ERD6-like 7 \\
FaSug11 & FANhyb_rscf00007047.1.g00001.1 & 1112 & 370 & sugar transport 10-like \\
FaSug12 & FANhyb_rscf00005538.1.g00001.1 & 1506 & 501 & sugar transport 5-like \\
FaSug13 & FANhyb_rscf00004023.1.g00001.1 & 1506 & 501 & sugar transport 5 \\
FaSug14 & FANhyb_icon00014335_a.1.g00001.1 & 1560 & 519 & sugar carrier C \\
FaSug15 & FANhyb_rscf00000458.1.g00001.1 & 1461 & 486 & sugar carrier C-like \\
\hline
\end{tabular}

bp - base pair, aa - amino acid. *BLAST search against NCBI NR database using BLAST2GO.

in contrast, the expression of the gene decreased with fruit maturation stages in low sugar containing cultivars (Fig. 1). The FaFrul showed lower expression in high sugar content cultivars and it's expression remained same in different stages (e.g., green, white and ripe stages) of fruit development. In contrast, this gene showed increasingly higher expression along with the progressive developmental stages of fruit in low sugar content cultivars. The expression FaFru2 decreased with the different stages of the fruit development in both high and low sugar content cultivars. The gene FaFru6 showed similar patterns of expression in all four cultivars (Fig. 1).

Expression profiling of Glucose related genes

Among 16 glucose related genes, six genes were selected based on their expression in four cultivars (Fig. 2). Like the fructose related genes, the glucose related genes also 


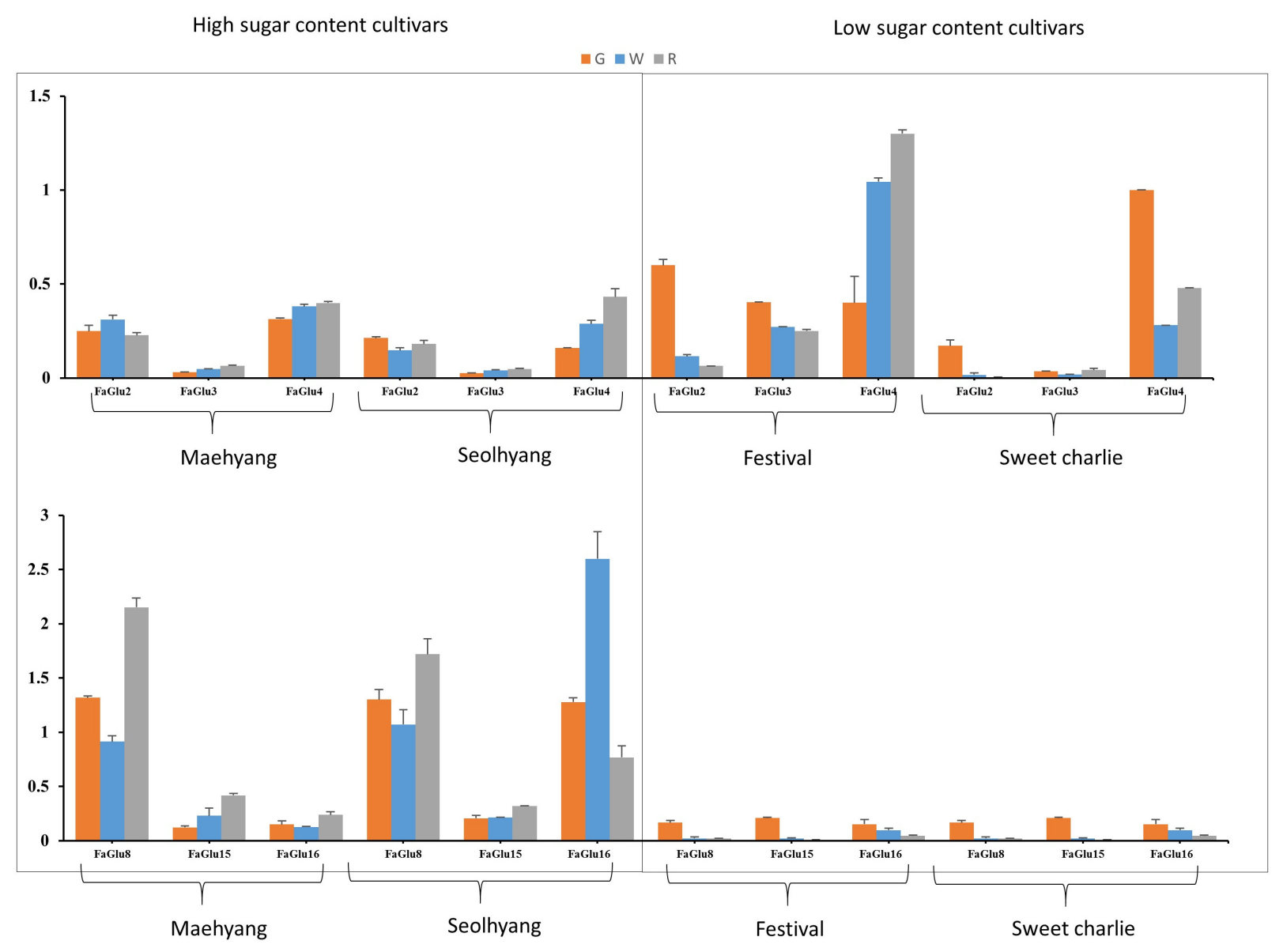

Fig. 2 Expression profiling of glucose related genes in contrasting strawberry cultivars

showed variable expression in different fruit developmental stages of the four studied cultivars (Fig. 2). The gene FaGlu3 showed contrastingly higher and lower expression along the progressive fruit developmental stages in high and low sugar containing cultivars, respectively. Its expression increased in high sugar containing cultivars whereas it decreased in low sugar containing cultivars along the fruit maturation stages. The expression of the gene FaGlu2 decreased with the progressive fruit developmental stages in all four cultivars. The expressions of the genes FaGlu8, FaGlu15 and FaGlu16 were very low in low sugar content cultivars compared to that of in high sugar containing cultivars (Fig. 2).

\section{Expression profiling of Sucrose related genes}

Among 18 sucrose related genes, eight genes were considerably expressed in all strawberry cultivars (Fig. 3). However, these genes showed higher expression in high sugar content cultivars compared to the low sugar content cultivars. The genes FaSuc1 and FaSuc11 showed increasingly higher expression whereas the FaSuc7 showed decreasing patterns of expression in high sugar content cultivars along the fruit maturation stages. However, rest of the genes showed unique expression between two high sugar content cultivars (Fig. 3).

Expression profiling of soluble sugar-related genes

Among 15 soluble sugar-related genes, seven were considerably expressed in four cultivars (Fig. 4). The genes FaSug10, FaSug12 and FaSug14 showed contrasting patterns of expression between the high and low sugar containing cultivars where the expression increased with the progress of fruit development in high sugar cultivars whereas decreased in low sugar cultivars (Fig. 4). The genes FaSug2, FaSug5 and FaSug7 showed decreasing patterns of expression along the development of fruit in all four cultivars.

\section{Discussion}

Strawberry fruits are mainly preferred for their characteristic flavor and color (besides it's nutritional value) around the world. Sweetness is one of the most important quality traits of strawberry that determines the consumer's pre- 


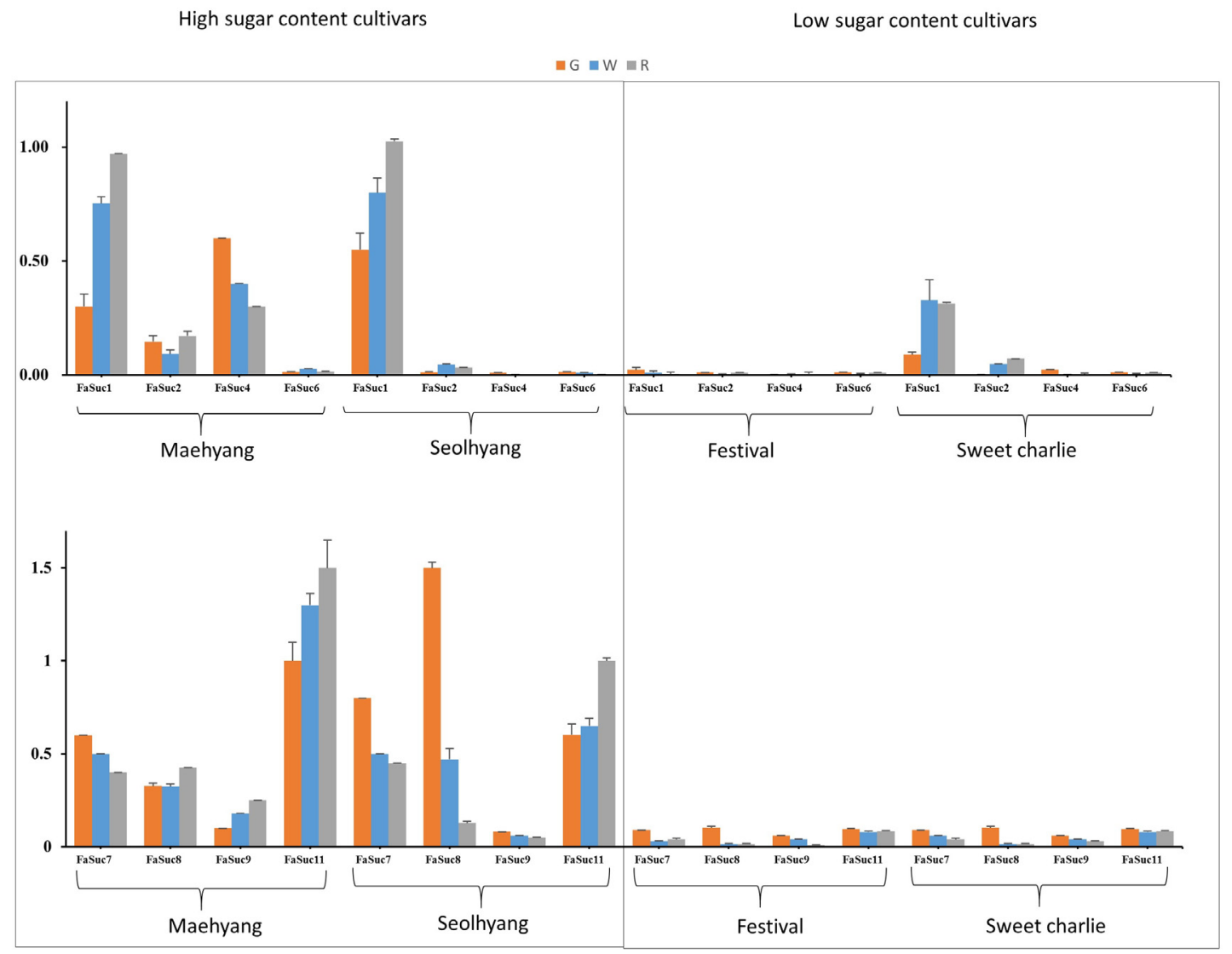

Fig. 3 Expression profiling of sucrose related genes in contrasting strawberry cultivars

High sugar content cultivars

Low sugar content cultivars

$\square \mathrm{G}=\mathrm{W}=\mathrm{R}$
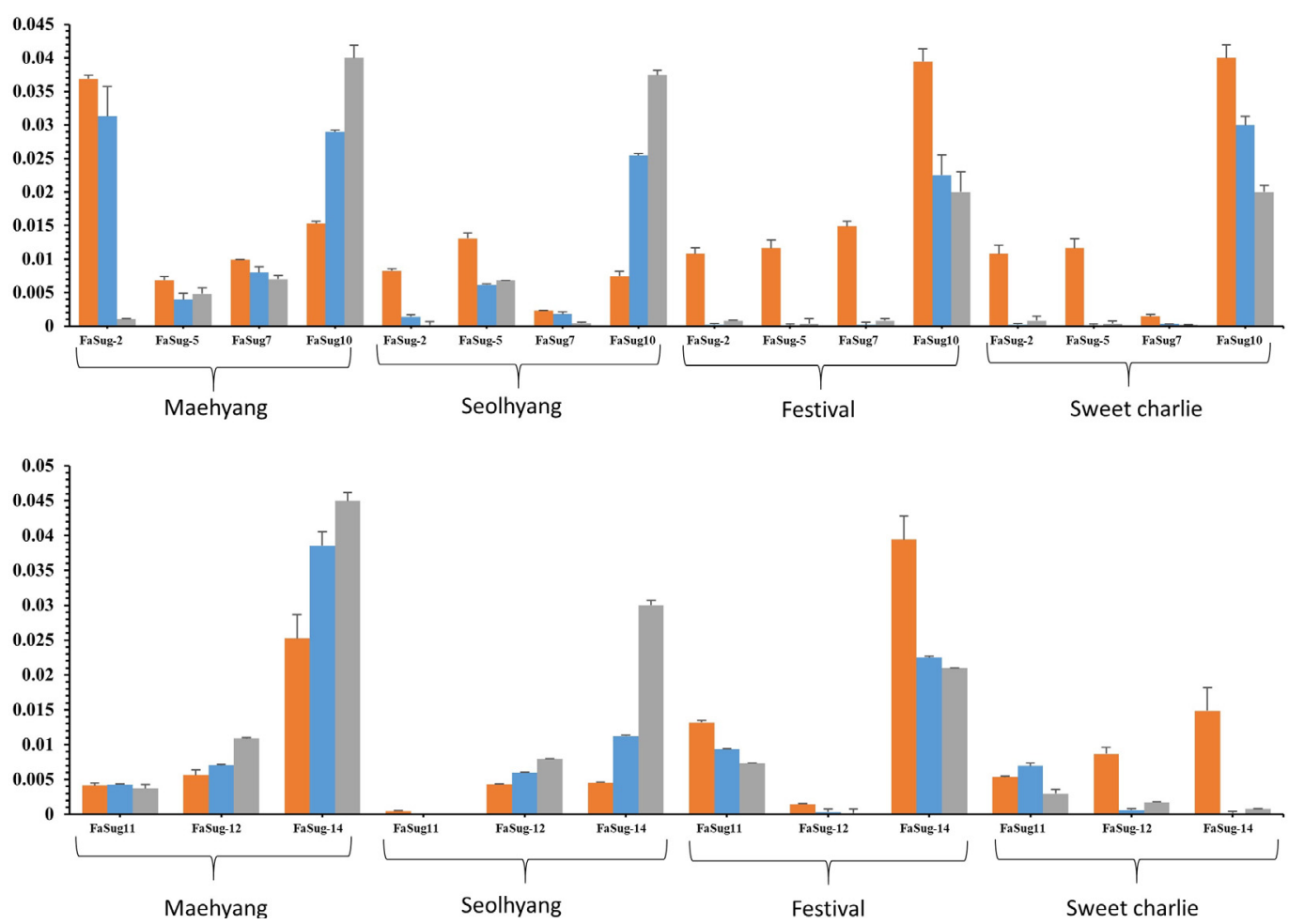

Fig. 4 Expression profiling of soluble sugar related genes in contrasting strawberry cultivars 
ference (Sturm et al. 2003; Gündüz and Özdemir 2014). The need for developing high sugar containing strawberry cultivars are thus of paramount importance to breeders. Understanding the role of the associated genes that regulates the biosynthesis of sugar is thus one of the key research areas in a way to identify the novel candidates for potential points of intervention in breeding programs. We analyzed the sugar content in four cultivars namely Maehyang, Seolhyang, Festival and Sweet Charlie and identified that first two are high sugar content and the second two are low sugar containing. This finding is in substantiated with the previous report of Kim et al.., 2009. The sugar profiles of these four genotypes were dominated by sucrose content and there is marked differences among the high vs low sugar content genotypes. For example, sucrose contents in both high sugar content genotypes Maehyang and Seolhyang are almost six times higher than that of the low sugar content genotypes Festival and Sweet Charlie (Table 2). However, no such obvious differences is observed between the low and high sugar content genotypes for Fructose and Glucose content making sucrose as the most important sugar to study the variations in sugar content in strawberry fruit. In strawberry, the variation in sugar and carbohydrate levels across genotypes and fruit developmental stages are diverge which can be pertained to the complexity of the sugar metabolism (Kafkas et al. 2007; Crespo et al. 2010; Mishra et al. 2015). The high sugar content in strawberry cultivars are generally responsible for its characteristic sweet flavor which increases the consumer preference and hence, the market value (Crespo et al. 2010).

Mechanism of fruit ripening is the complex process, which requires numerous fruit ripening and sugar related genes. These genes regulates the sugar metabolism, biosynthesis of hormones and ripening during the fruit development and growth (Jia et al. 2016; Zhongjie et al. 2016). Identifying the genes related to biosynthesis of total soluble sugars, glucose, sucrose and fructose would be helpful to widen our understanding on the variation in the biosynthesis of sugar in different cultivars and to identify the novel candidates as points of interventions for breeding programs. With this focus, we have mined the Strawberry garden database (http://strawberry-garden.kazusa.or.jp/) and identified 55 genome wide sugar related genes. Expression profiling of these genes via quantitative real-time PCR revealed that the expression of these sugar related genes vary during different stages of fruit development in the high and low sugar containing cultivars.

Most of the glucose and sucrose related genes were downregulated or showed lower expression in low sugar content cultivar compared to high sugar content cultivar, which might be responsible for sugar regulation in strawberry fruit in accordance with fruit development (Sturm et al. 2003; Basson et al. 2010; Jia et al. 2013). The expression profiling showed the considerable differential expression of glucose, sucrose and total soluble sugar related between contrasting cultivars based on sugar content. Among the genes involved in fructose biosynthesis, only the gene FaFru3 showed contrasting patterns of expression between high and low sugar-content cultivars across the fruit developmental stage in high and low sugar containing cultivars. The increasing patterns of expression in the progressive fruit developmental stages in the high sugar containing cultivars (and the decrease in the expression of this genes in low sugar containing cultivars) is certainly linked with the higher content of sugar in cultivars Mayhyang and Seolhyang. This makes it one of the candidate for further studying the fructose content in these genotypes. Similarly, the increasingly higher expression in high sugar containing cultivars and decreasing expression in low sugar containing cultivars along the fruit maturation stages of the glucose related gene FaGlu3 makes it the most important candidate for high sugar content in strawberry.

Among the various sugar compounds, sucrose dominated the sugar profile and thus genes that are involved in sucrose content will be very important candidate for any breeding program to develop high sugar containing strawberry cultivars (Jia et al. 2013; Jia et al. 2016). Among the 18 sucrose related genes studied, eight genes showed higher expression in high sugar content cultivars compared to the low sugar content cultivars. The genes FaSuc11 showed increasingly higher expression whereas the FaSuc7 showed decreasing patterns of expression in high sugar content cultivars along the fruit, maturation stages making this gene the most important candidate for sucrose content in these genotypes. The genes thus identified will serve as the target for any strawberry improvement programs for sugar contents.

\section{Conclusion}

From this study, we determined the fructose, sucrose and glucose content in contrasting strawberry cultivars. These sugars are responsible for the sweet flavor and quality of strawberry fruit. Among three sugars, sucrose is highly influencing the sweetness of strawberry fruit. Expression profiling of respective sugar related genes are substantiated their role in regulation of different sugar contents in contrasting strawberry cultivars. Expression profiling of respective 
sugar related genes substantiated their role in regulation of different sugars in relation with the sugar content. From the expression profiling, we find out some higher differentially expressed genes namely FaFru3, FaSuc11 and FaGlu8. Further, analysis and application of these genes will be helpful for marker-assisted selection followed by development of high sugar content cultivars.

\section{Acknowledgement}

This work was supported by grants from the Export Promotion Technology Development research programs (315047-3) funded by the Ministry of Agriculture, Food and Rural Affairs, Republic of Korea. Plant materials were received from Chungnam Agricultural Research \& Extension, South Korea.

\section{Conflict of Interest}

The authors declare that there are no conflicts of interest exists.

\section{Reference}

Amil-Ruiz F, Garrido-Gala J, Blanco-Portales R, et al. (2013) Identification and validation of reference genes for transcript normalization in strawberry (Fragaria $\times$ ananassa) defense responses. PLoS One 8:e70603

Basson CE, Groenewald JH, Kossmann J, et al. (2010) Sugar and acid-related quality attributes and enzyme activities in strawberry fruits: Invertase is the main sucrose hydrolysing enzyme. Food Chem 121:1156-1162

Basu A, Nguyen A, Betts NM, Lyons TJ (2014) Strawberry As a Functional Food: An Evidence-Based Review. Crit Rev Food Sci Nutr 54:790-806

Bestfleisch M, Möhring J, Hanke MV, et al. (2014) A diallel crossing approach aimed on selection for ripening time and yield in breeding of new strawberry (Fragaria $\times$ ananassa Duch.) cultivars. Plant Breed 133:115-120

Crespo P, Giné Bordonaba J, Terry LA, Carlen C (2010) Characterisation of major taste and health-related compounds of four strawberry genotypes grown at different Swiss production sites. Food Chem 122:16-24

Galli V, Borowski JM, Perin EC, et al. (2015) Validation of reference genes for accurate normalization of gene expression for real time-quantitative PCR in strawberry fruits using different cultivars and osmotic stresses. Gene 554:205-214

Giampieri F, Alvarez-Suarez JM, Mazzoni L, et al. (2013) The potential impact of strawberry on human health. Nat Prod Res 27:448-455

Gündüz K, Özdemir E (2014) The effects of genotype and growing conditions on antioxidant capacity, phenolic compounds, organic acid and individual sugars of strawberry. Food Chem 155:298-303

Jia H, Jiu S, Zhang C, et al. (2016) Abscisic acid and sucrose regulate tomato and strawberry fruit ripening through the abscisic acid-stress-ripening transcription factor. Plant Biotechnol J 14:2045-2065

Jia H, Wang Y, Sun M, et al. (2013) Sucrose functions as a signal involved in the regulation of strawberry fruit development and ripening Sucrose functions as a signal involved in the regulation of strawberry fruit development and ripening. New Phytol 453-465

Kafkas E, Koşar M, Paydaş S, et al. (2007) Quality characteristics of strawberry genotypes at different maturation stages. Food Chem 100:1229-1236

Kallio H, Hakala M, Pelkkikangas M, Lapvetel. (2000) Sugars and acids of strawberry varieties. Eur Food Res Technol 212:81-85

Kim DaeYoung; Yoon MooKyung; Kwak JungHo; Kim TaeIl and Kim JinHan (2009) Classification of strawberry germplasms based on horticultural traits and principal component analysis. Korean J Hortic Sci Technol 27:636-643

Macías-Rodríguez L, Quero E, López MG (2002) Carbohydrate differences in strawberry crowns and fruit (Fragaria x ananassa) during plant development. J Agric Food Chem 50:3317-21

Marchler-Bauer A, Derbyshire MK, Gonzales NR, et al. (2015) CDD: NCBI's conserved domain database. Nucleic Acids Res 43:D222-D226

Mishra PK, Ram RB, Kumar N, et al. (2015) Physico-chemical characteristics of some strawberry (Fragaria $x$ ananassa Duch.) genotypes. Intern ional J Multidiscip Res Dev Vol 2:216-218

Molina-Hidalgo FJ, Franco AR, Villatoro C, et al. (2013) The strawberry (Fragaria $\times$ ananassa) fruit-specific rhamnogalacturonate lyase 1 (FaRGLyase1) gene encodes an enzyme involved in the degradation of cell-wall middle lamellae. J Exp Bot 64:1471-1483

Natarajan S, Kim H, Thamilarasan SK, et al. (2016) Whole Genome Re-Sequencing and Characterization of Powdery Mildew Disease-Associated Allelic Variation in Melon. PLoS One 11:e0157524

Shanmugam A, Thamilarasan SK, Park J-I, et al. (2016) Characterization and abiotic stress-responsive expression analysis of SGT1 genes in Brassica oleracea. Genome 59:243-251

Sturm K, Koron D, Stampar F (2003) The composition of fruit of different strawberry varieties depending on maturity stage. Food Chem 83:417-422

Vijayakumar H, Thamilarasan SK, Shanmugam A, et al. (2016) Glutathione Transferases Superfamily: Cold-Inducible Expression of Distinct GST Genes in Brassica oleracea. Int J Mol Sci 17:1211

Wang, Shiow Y., Lin H-S (2000) Antioxidant activity in fruits and leaves. J Agric Food Chem 48:140-146

Zhongjie LIU, Pengcheng Z, Liwen CUI, et al. (2016) Over-expression of Gene FaASR Promotes Strawberry Fruit Coloring. Hortic Plant J 1:147-154 